\title{
Hermite collocation method for fractional order differential equations
}

\author{
Nilay Akgönüllü Pirim and Fatma Ayaz* \\ Department of Mathematics, Gazi University, Ankara, Turkey \\ nilayakgonullu@gmail.com, fayaz@gazi.edu.tr
}

\section{ARTICLE INFO}

\section{Article History:}

Received 03 May 2018

Accepted 13 July 2018

Available 20 July 2018

Keywords:

Fractional order differential equations

Hermite polynomials

Collocation methods

AMS Classification 2010:

26A33,33C45,35C10

\begin{abstract}
This paper focuses on the approximate solutions of the higher order fractional differential equations with multi terms by the help of Hermite Collocation method (HCM). This new method is an adaptation of Taylor's collocation method in terms of truncated Hermite Series. With this method, the differential equation is transformed into an algebraic equation and the unknowns of the equation are the coefficients of the Hermite series solution of the problem. This method appears as an useful tool for solving fractional differential equations with variable coefficients. To show the pertinent feature of the proposed method, we test the accuracy of the method with some illustrative examples and check the error bounds for numerical calculations.
\end{abstract}

\section{Introduction}

In many branches of science, mathematical models of physical processes require differential equations and nowadays, it is verified that some of these models can be better defined by fractional order equations due to the material and hereditary properties. Consequently, too many applications of the fractional order differential equations exist (see [1-5]). Unfortunately, model equations are usually in complex nature and involves nonlinear terms, therefore, analytical solutions can not easily be obtained. As a result, we still need more powerful numerical or approximate methods. Nowadays, many researchers are studying on numerical or approximate solutions of the fractional order equations and some new techniques have been introduced or adopted with the existent ones for ordinary case. For instance, finite difference 6-10], fractional linear multistep methods [11 13], Adomian decomposition [14 16], variational iteration method [16 18, differential transform or Taylor collocation method [19, 20] and spectral method [21 24] can be cited here. For some classes of fractional differential equations, Kumar and Agarwal mentioned about polynomial approximation methods and detailed information can be found in 25-27. There are also some other studies which worth to cite here [28 30. These are some valuable studies on fractional partial differential equations. In the recent years, many works have also been published on solving fractional differential equations but most of them have been concerned with a single term and the order is less than one. However, here, we adopt the Hermite Collocation method (HCM) for obtaining solutions to higher order multi-term fractional differential equations with variable coefficients. This technique evaluates an analytical solution in the form of a truncated Hermite series with unknown coefficients. In many physical problems, orthogonal functions or polynomials are used as a basis for obtaining solutions to the problems. On the other hand, the orthogonal Hermite polynomials are extensively 
used in some problems of hydrodynamics and meteorology [31]. The present method uses Hermite polynomials smilar to the Taylor collocation method and so is called Hermite Collocation Method which was first developed for higher-order linear Fredholm integro differential equation 32. Using this method has advantages on some partical types of physical processes as we mentioned above.

The second section of this study involves preliminary definitions and related theorems of the fractional calculus. In section 3, we recall the fundamental properties of the Hermite series and making adaptation of the method to the fractional order equation. Section 4 deals with the error bounds for the calculations and the section 5 involves some illustrative examples. Finally, we conclude the research with some highlights.

\section{Preliminary information and notations}

We start with the definition of Caputo derivative which was first introduced by Caputo ( [33]). This

$$
{ }^{C} D_{a}^{\alpha} f(x)=\left\{\begin{array}{c}
0, \\
\frac{\Gamma(\beta+1)}{\Gamma(\beta+1-\alpha)}(x-c)^{\beta-\alpha},
\end{array}\right.
$$

Some properties of the Caputo derivative can be given as follows:

Lemma 1. [1] Let $\alpha>0$ and let $y \in L^{\infty}(a, b)$ or $C([a, b])$. Then, $\left({ }^{C} D_{a}^{\alpha} I^{\alpha} y\right)(x)=y(x)$, where $I^{\alpha}$ defines the integral operator.

Lemma 2. [1] Let $\alpha>0$ and $n=[\alpha]+1$ where $[\alpha]$ is the integer part of $\alpha$. If $y \in A C^{n}([a, b])$ or $y \in C^{n}([a, b])$, then

$\left(I^{\alpha C} D_{a}^{\alpha} y\right)(x)=y(x)-\sum_{k=0}^{n-1} \frac{y^{(k)}(a)}{k !}(x-a)^{k}$.

Theorem 1. 34] For every $\alpha, \beta \in \mathbb{R}_{+}$the following relation holds,

$$
{ }^{C} D_{a}^{\alpha C} D_{a}^{\beta} f(x)={ }^{C} D_{a}^{\alpha+\beta} f(x) .
$$

\section{Hermite-collocation method for fractional order differential equations}

This section deals with the establishment of the theory of HCM for solving following multi-term fractional differential equations with variable coefficients,

$$
\sum_{k=0}^{m} P_{k}(x)^{C} D_{a}^{k \alpha} y(x)=g(x),
$$

derivative is preferred by many researches to make it easier to incorporate the initial and boundary conditions to the problem. Therefore, all the derivatives will be defined as Caputo derivatives throughout this study.

Definition 1. [1] Let $f \in A C^{n}[a, b]$ then, the Caputo fractional derivative of a function $f$ of order $\alpha>0$ is defined by

$$
\left({ }^{C} D_{a}^{\alpha} f\right)(x)=\frac{1}{\Gamma(n-\alpha)} \int_{a}^{x}(x-t)^{n-\alpha-1}\left(\frac{d}{d t}\right)^{n} f(t) d t
$$

where $\Gamma$ is the gamma function and $n-1<\alpha<$ $n, n \in N$.

Additionally, we can state the Caputo fractional derivative of a power function as follows. Let $\alpha \geq 0$, and $f(x)=(x-c)^{\beta}$ for some $\beta \geq 0$. Letting $c$ is any number, then

where $a \leq x \leq b, n-1<m \alpha<n(0<\alpha<1)$, $n>1, n \in \mathbf{N}, P_{k}(x)$ and $g(x)$ continuous on $a \leq$ $x \leq b$. Initial conditions are:

$$
{ }^{C} D^{j} y(a)=\lambda_{j}, j=0,1,2, \ldots, m \alpha-1 .
$$

In Eqs.(3)-(44), $D_{a}^{\alpha}$ or, for convenience $D^{\alpha}$ defines the Caputo derivative of order $\alpha$ and , $m \alpha-1$ is an integer number. We approximate the solution of the form as the following truncated Hermite series,

$$
y(x)=\sum_{k=0}^{N} a_{k} H_{k}\left(x^{\alpha}\right),
$$

where $a_{k}$ are unknown Hermite coefficients and $N \in \mathbf{N}^{+}$which satisfies $N \geq m \alpha$. To obtain the solution of Eq.(3) of the form Eq.(5), we first define the collocation points as $x_{i}=a+\left(\frac{b-a}{N}\right) i($ $i=0,1,2, \ldots N$, and $\left.x_{0}=a, x_{N}=b\right)$. Now, letting that $H\left(x^{\alpha}\right)=\left[H_{0}\left(x^{\alpha}\right) H_{1}\left(x^{\alpha}\right) H_{2}\left(x^{\alpha}\right) \ldots H_{N}\left(x^{\alpha}\right)\right]$ , $A=\left[\begin{array}{lll}a_{0} & a_{1} & a_{2} \ldots a_{N}\end{array}\right]^{T}$ then, Eq.(15) is written in matrix form as follow:

$$
[y(x)]=H\left(x^{\alpha}\right) A .
$$


Eventually, at collocation points, Eq.([6) is shown by $\left[y\left(x_{i}\right)\right]=H\left(x_{i}^{\alpha}\right) A$.

\subsection{Fractional hermite collocation method}

The Hermite polynomials of degree $\mathrm{n}$ are generated by the very well known formula, $H_{n}(x)=\sum_{n=0}^{\left[\left|\frac{n}{2}\right|\right]}(-1)^{m} \frac{n !}{(n-2 m) ! m !} x^{n-2 m}-\infty<x<$
$\infty, n=0,1,2 \ldots, N$.

Now we can define them in matrix notation (see [32])as below. If $N$ is an odd number, then the matrix notation of Hermite polynomials is written as

$$
\underbrace{\left[\begin{array}{c}
H_{0}\left(x^{\alpha}\right) \\
H_{1}\left(x^{\alpha}\right) \\
\vdots \\
H_{N-1}\left(x^{\alpha}\right) \\
H_{N}\left(x^{\alpha}\right)
\end{array}\right]}_{H^{T}\left(x^{\alpha}\right)}=\underbrace{\left[\begin{array}{ccccc}
2^{0} & 0 & \cdots & 0 & 0 \\
0 & 2^{1} & \cdots & 0 & 0 \\
\vdots & \vdots & \vdots & \vdots & \vdots \\
(-1)^{\left(\frac{N-5}{2}\right)} \frac{2^{0}}{0 !} \frac{(N-1) !}{\left(\frac{N-1}{2}\right) !} & 0 & \cdots & 2^{N-1} & 0 \\
0 & (-1)^{\left(\frac{N-1}{2}\right)} \frac{2^{1}}{1 !} \frac{N !}{\left(\frac{N-1}{2}\right) !} & \cdots & 0 & 2^{N}
\end{array}\right]}_{F} \underbrace{\left[\begin{array}{c}
1 \\
x^{\alpha} \\
\vdots \\
x^{\alpha(N-1)} \\
x^{\alpha N}
\end{array}\right]}_{X^{T}\left(x^{\alpha}\right)}
$$

if $N$ is even then it follows,

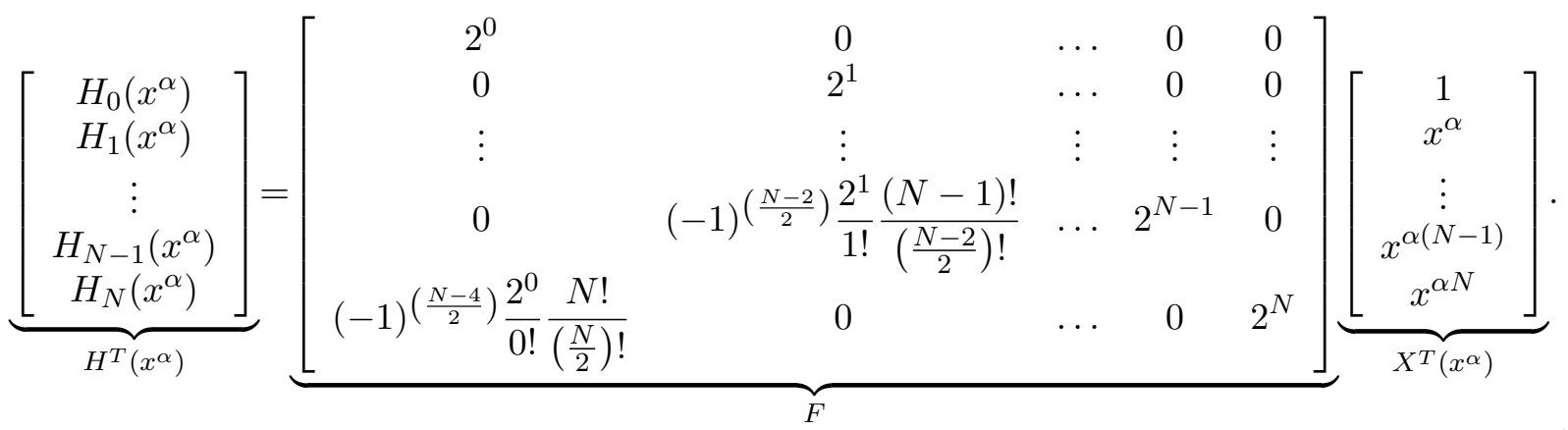

Consequently, we can write the above matrices shortly,

$$
y(x)=X\left(x^{\alpha}\right) F^{T} A .
$$

$$
H^{T}\left(x^{\alpha}\right)=F X^{T}\left(x^{\alpha}\right)
$$

\subsection{Caputo derivatives of operational matrix}

Now, we need to determine any $k \alpha$ th order Ca-

or

$$
H\left(x^{\alpha}\right)=X\left(x^{\alpha}\right) F^{T}
$$
puto fractional derivatives of Eq.(10) by the following procedure,

$$
{ }^{C} D^{k \alpha} y(x)={ }^{C} D^{k \alpha} X\left(x^{\alpha}\right) F^{T} A .
$$

More generally, if we show that

$$
\begin{array}{r}
{ }^{C} D^{k \alpha} X\left(x^{\alpha}\right)=\left[{ }^{C} D^{k \alpha}(x-c)^{0}{ }^{C} D^{k \alpha}(x-c)^{1 \alpha}\right. \\
\left.\ldots{ }^{C} D^{k \alpha}(x-c)^{(N-1) \alpha C} D^{k \alpha}(x-c)^{N \alpha}\right]
\end{array}
$$

then, the substitution of Eq.(91) into Eq.(6) yields,

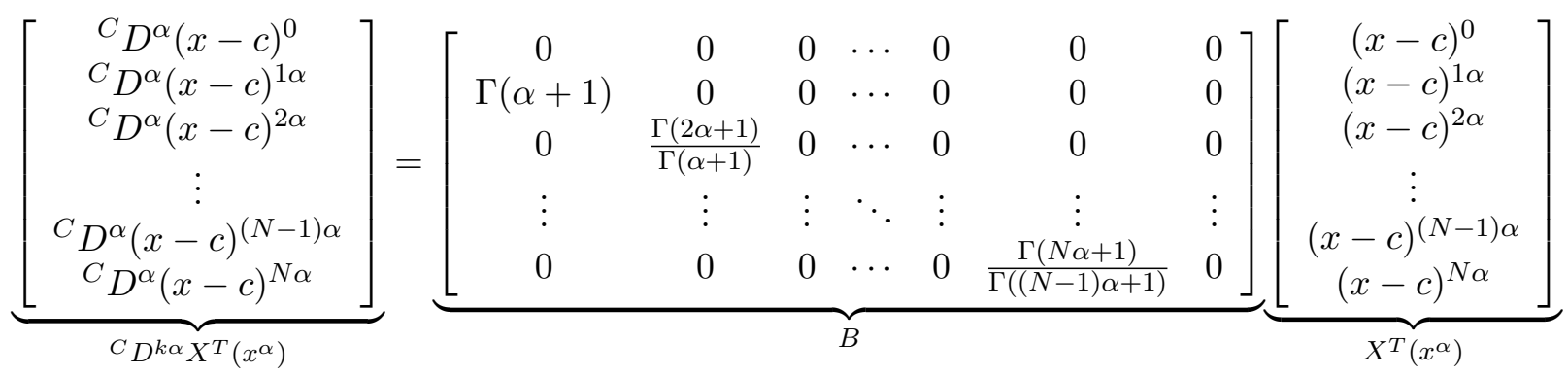


Consequently, one can write that ${ }^{C} D^{\alpha} X\left(x^{\alpha}\right)=$ $X\left(x^{\alpha}\right) B^{T}$ and the following theorem holds.

Theorem 2. Let $X\left(x^{\alpha}\right)$ be the Hermite polynomial vector, for any $\alpha>0$, then we have,

$$
{ }^{C} D^{k \alpha} y(x)=X\left(x^{\alpha}\right)\left(B^{T}\right)^{k} F^{T} A,
$$

Proof. By the help of Theorem 1, the successive $\alpha$ th order Caputo fractional derivatives of $X\left(x^{\alpha}\right)$ become,

$$
\begin{gathered}
{ }^{C} D^{\alpha C} D^{\alpha} X\left(x^{\alpha}\right)=\underbrace{{ }^{C} D^{\alpha} X\left(x^{\alpha}\right)}_{X\left(x^{\alpha}\right) B^{T}} B^{T}, \\
{ }^{C} D^{2 \alpha} X\left(x^{\alpha}\right)=X\left(x^{\alpha}\right)\left(B^{T}\right)^{2}, \\
\vdots \\
{ }^{C} D^{k \alpha} X\left(x^{\alpha}\right)=X\left(x^{\alpha}\right)\left(B^{T}\right)^{k} .
\end{gathered}
$$

Hence, substitution of Eq.(13) into Eq.(11) gives,

$$
{ }^{C} D^{k \alpha} y(x)=X\left(x^{\alpha}\right)\left(B^{T}\right)^{k} F^{T} A .
$$

Eq.(14), is also shown by the following formula at collocation points $x=x_{i}$ as,

$$
{ }^{C} D^{k \alpha} y\left(x_{i}\right)=X\left(x_{i}^{\alpha}\right)\left(B^{T}\right)^{k} F^{T} A .
$$

Now, let recall the differential equation redefined at collocation points as same as below,

$$
\sum_{k=0}^{m} P_{k}\left(x_{i}\right)^{C} D^{k \alpha} y\left(x_{i}\right)=g\left(x_{i}\right), \quad i=0,1,2, \ldots, N,
$$

therefore, Eq.(16) is written in the following matrix form:

$$
\begin{gathered}
\underbrace{\left[\begin{array}{cccc}
P_{k}\left(x_{0}\right) & 0 & \cdots & 0 \\
0 & P_{k}\left(x_{1}\right) & \cdots & 0 \\
\vdots & \vdots & \ddots & \vdots \\
0 & 0 & \cdots & P_{k}\left(x_{N}\right)
\end{array}\right]}_{P_{k}} \\
\times \underbrace{\left[\begin{array}{c}
{ }^{C} D^{k \alpha} y\left(x_{0}\right) \\
{ }^{C} D^{k \alpha} y\left(x_{1}\right) \\
\vdots \\
{ }^{C} D^{k \alpha} y\left(x_{N}\right)
\end{array}\right]}_{Y^{k \alpha}}=\underbrace{\left[\begin{array}{c}
g\left(x_{0}\right) \\
g\left(x_{1}\right) \\
\vdots \\
g\left(x_{N}\right)
\end{array}\right]}_{G} .
\end{gathered}
$$

In the compact form, Eq.(16) can be given as,

$$
\sum_{k=0}^{m} P_{k} Y^{k \alpha}=G
$$

On the other hand, we have by Eq.(15),

$$
\underbrace{\left[\begin{array}{c}
{ }^{C} D^{k \alpha} y\left(x_{0}\right) \\
{ }^{C} D^{k \alpha} y\left(x_{1}\right) \\
\vdots \\
{ }^{C} D^{k \alpha} y\left(x_{N}\right)
\end{array}\right]}_{Y^{k \alpha}}=\underbrace{\left[\begin{array}{c}
X\left(x_{0}^{\alpha}\right) \\
X\left(x_{1}^{\alpha}\right) \\
\vdots \\
X\left(x_{N}^{\alpha}\right)
\end{array}\right]}_{X^{\alpha}}\left[\left(B^{T}\right)^{k} F^{T} A\right]
$$

where the matrix $X^{\alpha}$ is equivalent to

$$
X^{\alpha}=\underbrace{\left[\begin{array}{c}
X\left(x_{0}^{\alpha}\right) \\
X\left(x_{1}^{\alpha}\right) \\
\vdots \\
X\left(x_{N}^{\alpha}\right)
\end{array}\right]}_{X^{\alpha}}=\left[\begin{array}{ccccc}
1 & \left(x_{0}-c\right)^{1 \alpha} & \cdots & \left(x_{0}-c\right)^{(N-1) \alpha} & \left(x_{0}-c\right)^{N \alpha} \\
1 & \left(x_{1}-c\right)^{1 \alpha} & \cdots & \left(x_{1}-c\right)^{(N-1) \alpha} & \left(x_{1}-c\right)^{N \alpha} \\
\vdots & \vdots & \ddots & \vdots & \vdots \\
1 & \left(x_{N}-c\right)^{1 \alpha} & \cdots & \left(x_{N}-c\right)^{(N-1) \alpha} & \left(x_{N}-c\right)^{N \alpha}
\end{array}\right] \text {. }
$$

Hence, we can rewrite Eq.(15) as follows,

$$
Y^{k \alpha}=X^{\alpha}\left(B^{T}\right)^{k} F^{T} A .
$$

Finally, the substitution Eq.(18) into Eq. (17) gives the fundamental matrix equation such as

$$
\sum_{k=0}^{m} P_{k} X^{\alpha}\left(B^{T}\right)^{k} F^{T} A=G .
$$

Moreover, denoting

$$
W=\sum_{k=0}^{m} P_{k}(x) X^{\alpha}\left(B^{T}\right)^{k} F^{T},
$$

where $W=\left[w_{i j}\right](i, j=0,1,2, \ldots N)$, then, Eq. (19) is shown by,

$$
W \cdot A=G .
$$

Now, Eq. (20) generates an algebraic system which consists of $(\mathrm{N}+1)$ rows and $(\mathrm{N}+1)$ columns. Then, the augmented matrix of the system is written by, 
$[W ; G]=\left[\begin{array}{cccccc}w_{00} & w_{01} & \cdots & w_{0 N} & ; & g\left(x_{0}\right) \\ w_{10} & w_{11} & \cdots & w_{1 N} & ; & g\left(x_{1}\right) \\ \vdots & \vdots & \vdots & \vdots & ; & \vdots \\ w_{(N-1) 0} & w_{(N-1) 1} & \cdots & w_{(N-1) N} & ; & g\left(x_{N-1}\right) \\ w_{N 0} & w_{N 1} & \cdots & w_{N N} & ; & g\left(x_{N}\right)\end{array}\right]$

This method can be modified to handle the initial conditions defined at particular point $a$. Therefore, we recall the initial Eq. (4),

$$
{ }^{C} D^{j} y(a)=\lambda_{j}, j=0,1,2, \ldots m \alpha-1 .
$$

Hence, substitution of these conditions into Eq.(15) yields,

$$
X^{\alpha}(a)\left(B^{T}\right)^{j} F^{T} A=\lambda_{j} .
$$

Therefore, defining $U_{j}$ as,

$U_{j}=X^{\alpha}(a)\left(B^{T}\right)^{j} F^{T}=\left[\begin{array}{lllll}u_{j 0} & u_{j 1} & u_{j 2} & \cdots & u_{j N}\end{array}\right]$

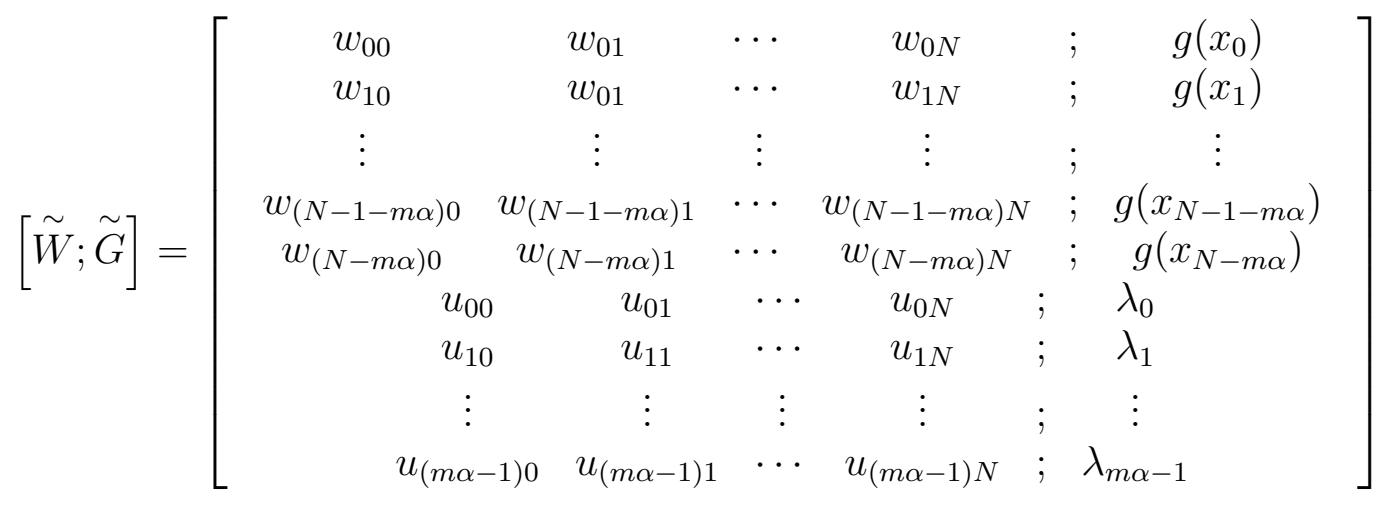

Hence, the system of algebraic equations are shown by the following notation,

$$
\tilde{W} A=\tilde{G} .
$$

Remark 1. Now, let us consider the system $\tilde{W} A=\tilde{G}$.

If $\operatorname{rank} \tilde{W}=\operatorname{rank}[\tilde{W}, \tilde{G}]=N+1$, (i.e $\operatorname{det}(\tilde{W}) \neq$ 0 ) then we can write

$$
A=(\tilde{W})^{-1} \tilde{G}
$$

Consequently, the Hermite coefficients $a_{k}(k=$ $0,1,2, \ldots, N)$ can be uniquely determined by Eq.(25). As a result, the truncated Hermite series is written as follows, then, Eq.(22) can be shown by

$$
U_{j} A=\lambda_{j},
$$

and corresponding augmented matrix is written of the form,

$$
\left[U_{j} ; \lambda_{j}\right], j=0,1,2, \ldots, m \alpha-1
$$

and denoted by

$\left[U_{j} ; \lambda_{j}\right]=\left[\begin{array}{cccccc}u_{00} & u_{01} & \cdots & u_{0 N} & ; & \lambda_{0} \\ u_{10} & u_{01} & \cdots & u_{1 N} & ; & \lambda_{1} \\ \vdots & \vdots & \vdots & \vdots & ; & \vdots \\ u_{(m \alpha-1) 0} & u_{(m \alpha-1) 1} & \cdots & u_{(m \alpha-1) N} & ; & \lambda_{m \alpha-1}\end{array}\right]$

Now, if the math row of the the augmented matrix Eq.(21) of the system is replaced by the augmented matrix of initial conditions Eq.(24), then one can write the following matrix form,

$$
y(x)=\sum_{k=0}^{N} a_{k} H_{k}\left(x^{\alpha}\right) .
$$

\section{Error bounds}

Eq.(27) is the approximate solution to Eq.(3) with the initial conditions, Eq.(41). Therefore, substitution the truncated Hermite series into the problem, we obtain the residuals;

$$
\left|\sum_{k=0}^{m} P_{k}\left(x_{i}\right)^{C} D^{k \alpha} y\left(x_{i}\right)-g\left(x_{i}\right)\right|
$$

at $x=x_{i} \quad(-\infty<a \leq x \leq b<\infty), i=$ $0,1,2, \ldots, N$. Then, we call the error function as $E\left(x_{i}\right)$ and this function should be less than $\epsilon$, which is a positive number and can arbitrarily 
be chosen as $10^{-k_{i} \alpha}$. As a result, error function becomes, $E\left(x_{i}\right) \leq 10^{-k_{i} \alpha}$ where $k_{i}>0$ is any constant. If the $\max \left(10^{-k_{i} \alpha}\right)=10^{-k \alpha}$ is desired accuracy then, the truncation limit $N$ is incerased untill $E\left(x_{i}\right)$ approaches zero. Besides, the global error function is defined as follows,

$$
E_{N}(x)=\sum_{k=0}^{m} P_{k}(x)^{C} D^{k \alpha} y(x)-g(x) .
$$

Consequently, the global error, $E_{N}(x) \rightarrow 0$ when $N$ is sufficiently large.

\section{Illustrative examples}

The method which was mentioned so far has been used to solve multi term fractional order differential equations. To show the accuracy of the method, the following examples have been solved. All the numerical calculations have been performed by using Matlab v7.5. and the results have been given by Figure 1 for different values of $\alpha$. The comparisons between exact and Hermite polynomial solution approximation have been made and shown by Table 1 .

Example 1. First we consider Bagley-Torvik equation [35];

$$
D^{2} y(x)+D^{3 / 2} y(x)+y(x)=x+1,
$$

where $\alpha=1 / 2, m=4$ and $g(x)=x+1$. To find HCM solution of the problem here, for conveniance, we choose $N=2$. Because the analytical solution of the problem for $\alpha=1$ is easily obtainable. Therefore, we only concentrate on the different values of $\alpha$. Hence, the approximate solution can be written by the following truncated Hermite series:

$$
y(x)=\sum_{n=0}^{2} a_{n} H_{n}\left(x^{\alpha}\right) .
$$

The coefficients of the differential equation are $P_{0}(x)=P_{3}(x)=P_{4}(x)=1, P_{1}(x)=P_{2}(x)=0$ Since $N=2$ then, collocation points are taken as $\left\{x_{0}=0, x_{1}=1 / 2, x_{2}=1\right\}$. From the fundamental matrix equation Eq.(19), one can write that

$$
\left\{P_{0} X+P_{3} X\left(B^{T}\right)^{3}+P_{4} X\left(B^{T}\right)^{4}\right\} F^{T} A=G .
$$

After evaluating the matrices $B$ and $F$ and substituting them into the above equation, then, the augmented matrix is obtained as follows,

$$
W . A=G \Rightarrow[W ; G]=\left[\begin{array}{ccccc}
1 & 0 & -2 & ; & 1 \\
1 & 1.4 & 0 & ; & 1.5 \\
1 & 2 & 2 & ; & 2
\end{array}\right]
$$

Since $\operatorname{det}(W) \neq 0$, finding the solution of the system defines the coefficients of the truncated series as

$$
\left[\begin{array}{l}
a_{0} \\
a_{1} \\
a_{2}
\end{array}\right]=\left[\begin{array}{c}
1.5 \\
0 \\
0.25
\end{array}\right]
$$

Finally, substituting these coefficients into $E q \cdot(29)$,

$$
y(x)=a_{0} H_{0}\left(x^{\alpha}\right)+a_{1} H_{1}\left(x^{\alpha}\right)+a_{2} H_{2}\left(x^{\alpha}\right)
$$

then, we obtain Hermite polynomial solution of the problem as

$$
y(x)=\frac{3}{2}+\frac{1}{4}\left(4 x^{\alpha}-2\right) .
$$

From here, if we substitute $\alpha=1$ in $y(x)$ then, we obtain $y(x)=x+1$. This is the exact solution of the problem for integer order case.

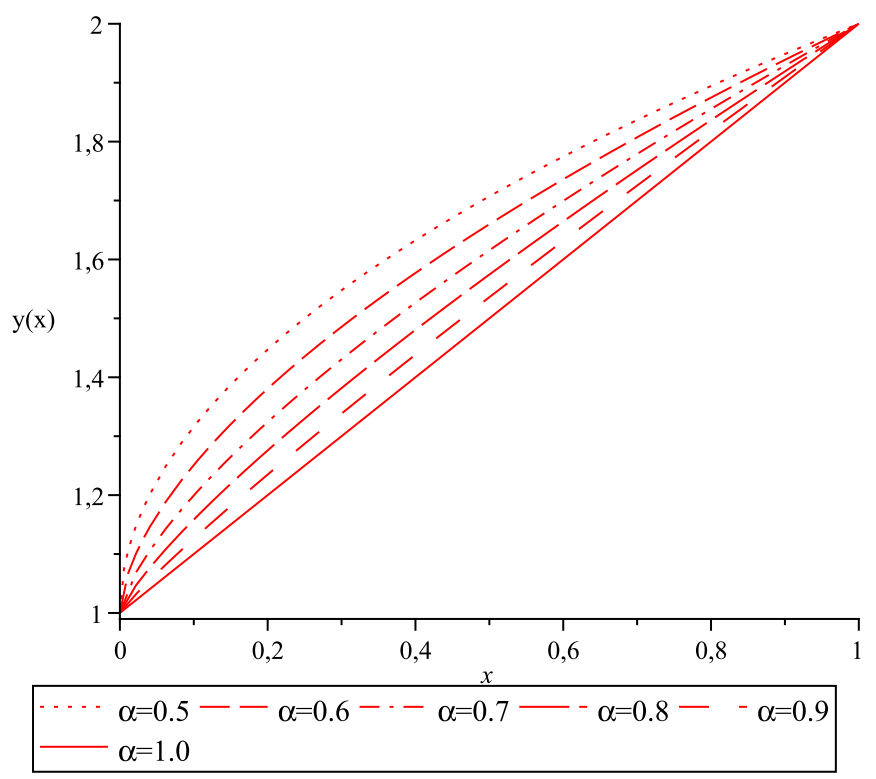

Figure 1. The solution curves of Example 1 for different values of $\alpha$. The $\alpha$ values change from 0.5 to 1 .

Example 2. Next we consider an initial value problem which is studied in [36] where $0 \leq x \leq 1$ and $\alpha \in(0,1), \beta \leq 0$. Therefore, we can write the equation as: 


$$
D^{\alpha} y(x)=\beta y(x)+g(x) .
$$

We assume that $\beta=-1$ and $g(x)=x^{2}+\frac{2 x^{2-\alpha}}{\Gamma(3-\alpha)}$ defined on $[0,1]$ and the initial condition is $y(0)=$ 0. If we apply the HCM for $\alpha=\frac{1}{2}$ and $N=4$, it is obvious that $m$ is 2 and $P_{0}(x)=P_{1}(x)=$
$1, P_{2}(x)=0$. Therefore, we can write the following fundamental matrix of Eq.(30) as,

$$
\left\{P_{0} X+P_{1} X B^{T}+P_{2} X\left(B^{T}\right)^{2}\right\} F^{T} A=G .
$$

Therefore, we can easily establish the system $W A=G$ and constitute $[W ; G]$ matrix as,

$$
[W ; G]=\left[\begin{array}{ccccccc}
1.0000 & 1.7725 & -2.0000 & -10.6347 & 12.0000 & ; & 0.0000 \\
1.0000 & 2.7725 & 1.2568 & -12.9760 & -23.0721 & ; & 0.2506 \\
1.0000 & 3.1867 & 3.1915 & -10.9742 & -37.7877 & ; & 0.7818 \\
1.0000 & 3.5045 & 4.9088 & -7.8548 & -46.2706 & ; & 1.5397 \\
1.0000 & 3.7725 & 6.5135 & -4.0000 & -50.0901 & ; & 0.0000
\end{array}\right]
$$

On the other hand, the augmented matrix, which corresponds to initial condition, is obtained by substitution the row,

$$
y(0)=X(\mathbf{0}) F^{T} A=\lambda_{0}=0,
$$

$$
y(0)=\left[\begin{array}{lllll}
1 & 0 & 0 & 0 & 0
\end{array}\right] F^{T} A=0 .
$$

Finally, we find that $\left[U_{0} ; \lambda_{0}\right]=$ $\left[\begin{array}{lllllll}1 & 0 & -2 & 0 & 12 & ; & 0\end{array}\right]$. Therefore, the augmented matrix of the system, $\widetilde{W} A=\widetilde{G}$, is obor tained from Eq.(25) as follows,

$$
[\tilde{W} ; \tilde{G}]=\left[\begin{array}{ccccccc}
1.0000 & 1.7725 & -2.0000 & -10.6347 & 12.0000 & ; & 0.0000 \\
1.0000 & 2.7725 & 1.2568 & -12.9760 & -23.0721 & ; & 0.2506 \\
1.0000 & 3.1867 & 3.1915 & -10.9742 & -37.7877 & ; & 0.7818 \\
1.0000 & 3.5045 & 4.9088 & -7.8548 & -46.2706 & ; & 1.5397 \\
1.0000 & 0.0000 & -2.0000 & 0.0000 & 12.0000 & ; & 0.0000
\end{array}\right]
$$

Consequently, solution of the above system gives the approximate solution of the problem as,

$y(x)=0,986076 x 10^{-31} x^{1 / 2}+0,104468 \times 10^{-13} x^{3 / 2}+x^{2}$.
We note here that our solution is very close to the exact solution, $y(x)=x^{2}$, since the first two terms vanishes (see Figure 2).

As a result of all these, the HCM solution is very good approximation to the problem even for small $N$. Table 1 lists both the exact solution and error function at particular $x$, corresponding to the Example.

Table 1. The exact solution of Example 2 and error function $E\left(x_{i}\right)$ for HCM solution.

\begin{tabular}{|c|c|c|}
\hline$x$ values & Exact Solution & $E\left(x_{i}\right)$ For HCM solution, $N=4$ \\
\hline 0.0 & 0.0000 & $9.0206 \mathrm{e}-17$ \\
\hline 0.2 & $4.0000 \mathrm{e}-2$ & $9.0206 \mathrm{e}-17$ \\
\hline 0.4 & $1.6000 \mathrm{e}-1$ & $2.7756 \mathrm{e}-16$ \\
\hline 0.6 & $3.6000 \mathrm{e}-1$ & $4.9960 \mathrm{e}-16$ \\
\hline 0.8 & $6.4000 \mathrm{e}-1$ & $7.7716 \mathrm{e}-16$ \\
\hline 1.0 & 1.0000 & $1.1102 \mathrm{e}-15$ \\
\hline
\end{tabular}

In Figure 2, both the analytical and the HCM solutions (for $N=2$ and $N=4$ ) are given. It is clear that the series (for $N=4$ ) and the analytical solution are identical. 


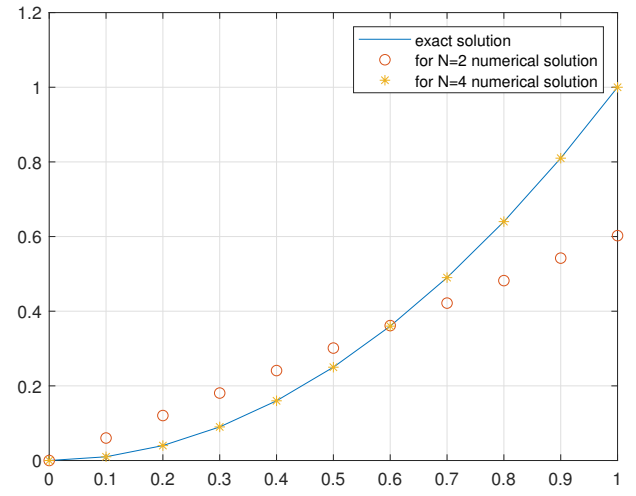

Figure 2. Comparison of Analytical solution and HCM solution for Example 2 .

\section{Conclusion}

The objective of this study is to apply the HCM method for solving higher order multi-term fractional order differential equations. The motivation of this work is that obtaining considerable simplifications in the solutions of the multi-term fractional order differential equations by using HCM, since the analytical solutions of such equations cannot easily be obtained. By using any symbolic toolbox of Matlab programme, the Hermite polynomial coefficients of the solution can be obtained easily. Illustrated examples determine the reliability of the algorithm and give chance to apply the method for wider classes of equations. As a further work, the method will be considered for solving nonlinear fractional order differential equations.

\section{References}

[1] Kilbas, A.A., Sirvastava, H.M., Trujillo, J.J. (2006). Theory and Application of Fractional Differential Equations. North-Holland Mathematics Studies. Vol 204, Amsterdam.

[2] Mainardi, F., Luchko, Yu., Pagnini, G. (2001). The fundamental Solution of the Space-Time Fractional Diffusion Equation. Frac. Calc. Appl. Anal., 4(2), 153152.

[3] Ross, B. (1975). Fractional Calculus and its Applications. Lecture Notes in Mathematics. Vol. 457, Springer Verlag.

[4] Stanislavsky, A.A. (2004) Fractional Oscillator. Phys. Rev. E., 70(5).

[5] Trujillo, J.J. (1999). On a Riemann-Liouville Generalised Taylor's Formula. J. Math. Anal. Appl., 231, 255-265.

[6] Podlubny, I. (1999). Fractional Differential Equations. Academic Press, New York.

[7] Ciesielski, M., Leszcynski, J. (2003). Numerical simulations of anomalous diffusion. Computer Methods Mech. Conference, Gliwice Wisla Poland.
[8] Yuste, S.B. (2006). Weighted average finite difference methods for fractional diffusion equations. J. Comput. Phys., 1, 264-274.

[9] Odibat, Z.M. (2006). Approximations of fractional integrals and Caputo derivatives. Appl. Math. Comput., 527-533.

[10] Odibat, Z.M. (2009). Computational algorithms for computing the fractional derivatives of functions. Math. Comput. Simul., 79(7), 2013-2020.

[11] Ford, N.J., Connolly, A.Joseph. (2009). Systemsbased decomposition schemes for the approximate solution of multi-term fractional differential equations. J. Comput. Appl. Math., 229, 382-391.

[12] Ford, N.J. (2001). Simpson, A. Charles, The numerical solution of fractional differential equations:speed versus accuracy. Numer. Alg., 26, 333-346.

[13] Sweilam, N.H., Khader, M.M., Al-Bar, R.F. (2007). Numerical studies for a multi order fractional differential equations. Phys. Lett. A, 371, 26-33.

[14] Momani, S., Odibat, Z. (2006). Analytical solution of a time-fractional Navier-Stokes equation by adomian decomposition method. Appl. Math. Comput., 177, 488-494.

[15] Odibat, Z., Momani, S. (2007). Numerical approach to differential equations of fractional order. J. Comput. Appl. Math., 207(1), 96-110.

[16] Odibat, Z., Momani, S. (2008). Numerical methods for nonlinear partial differential equations of fractional order. Appl. Math. Model., 32, 28-39.

[17] Odibat, Z., Momani, S. (2006). Application of variational iteration method to equation of fractional order. Int. J. Nonlinear Sci. Numer. Simul., 7, 271-279.

[18] Wu, J.L. (2009). A wavelet operational method for solving fractional partial differential equations numerically. Appl. Math. Comput., 214, 31-40.

[19] Ertürk,V.S., Momani, S. (2008). Solving systems of fractional differential equations using differential transform method. J. Comput. Appl. Math., 215, 142151.

[20] Yalçınbaş, S., Konuralp, A., Demir, D.D., Sorkun, H.H. (2010). The solution of the fractional differential equation with the generalized Taylor collocation method. IJRRAS, August 2010.

[21] Abd-Elhameed, W.M., Youssri, Y.H. (2016). A Novel Operational Matrix of Caputo Fractional Derivatives of Fibonacci Polynomials: Spectral Solutions of Fractional Differential Equations. Entropy, 18(10), 345.

[22] Abd-Elhameed, W.M., Youssri, Y.H. (2016). Spectral Solutions for Fractional Differential Equations via a Novel Lucas Operational Matrix of Fractional Derivatives. Romanian Journal of Physics, 61(5-6), 795-813.

[23] Abd-Elhameed, W.M., Youssri, Y.H., (2015). New Spectral Solutions of Multi-Term Fractional-Order Initial Value Problems With Error Analysis. CMES: Computer Modeling in Engineering and Sciences, 105(5), 375-398.

[24] Youssri, H., Abd-Elhameed W.M. (2016). Spectral Solutions for Multi-Term Fractional Initial Value Problems Using a New Fibonacci Operational Matrix of Fractional Integration. Progress in Fractional Differentiation and Applications, 2(2), 141-151.

[25] Kumar, P., Agrawal, O.P. (2006). An approximate method for numerical solution of fractional differential equations. Signal Process, 86, 2602-2610. 
[26] Kumar, P. (2006). New numerical schemes for the solution of fractional differential equations. Southern Illinois University at Carbondale. Vol 134.

[27] Kumar, P., Agrawal, O.P. (2006). Numerical scheme for the solution of fractional differential equations of order greater than one. J. Comput. Nonlinear Dyn., $1,178-185$.

[28] Yavuz M., Özdemir, N. (2018). A Different Approach to the European Option Pricing Model with New Fractional Operator. Mathematical Modelling of Natural Phenomena, 13(1),1-12.

[29] Avci, D., Eroglu İskender, B.B, Özdemir, N. (2017). Conformable heat equation on a radial symmetric plate. Thermal Science, 21(2), 819-826.

[30] Yavuz, M., Özdemir, N. (2018). Numerical inverse Laplace homotopy technique for fractional heat equations. Thermal Science, 22(1), 185-194.

[31] Dattoli, G. (2004). Laguerra and generalized Hermite polynomials: the point of view of the operation method. Integral Transforms Special Func., 15, 93-99.

[32] Akgönüllü, N.,Şahin, N., Sezer, M. (2010). A Hermite Collocation Method for the Approximate Solutions of Higher-Order Linear Fredholm Integro-Differential Equations. Numerical Methods for Partial Differential Eqautions, 27(6), 1708-1721.

[33] Caputo, M. (1967). Linear models of dissipation whose $\mathrm{Q}$ is almost frequency independent II. Geophys. $J$. Royal Astron. Soc., 13, 529-539.

\section{An International Journal of Optimization and Control: Theories \& Applications (http://ijocta.balikesir.edu.tr)}

[34] Samko,S., Kilbas, A., Marichev, O. (1993). Fractional integral and derivatives theory and applications. Gordon and Breach, New York.

[35] Diethelm, K., Ford, N.J. (2002). Numerical Solution of the Bagley-Torvik Equation. BIT, 42, 490-507.

[36] Diethelm K. (1997). An algorithm for the numerical solution of differential equations of fractional order. Electronic Transactions on Numerical Analysis,5, 1-6.

Fatma Ayaz is a Professor in the Department of Mathematics at Gazi University, Ankara, Turkey. She received her Ph.D. of Mathematics in Leeds University $(U K)$, 1995. She has many research papers about numerical solutions of differential equations and fractional ordinary differential equations, computational methods, computational fluid dynamics, mathematical modelling. She has supervised 14 graduate students and has been supervising 6 graduate students.

Nilay Akgönüllü Pirim received her PhD. degree in Mathematics from Gazi University in 2014. Her research interests focuses mainly on the approximate solutions of fractional differential equations and Hermite collocation Method.

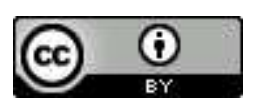

This work is licensed under a Creative Commons Attribution 4.0 International License. The authors retain ownership of the copyright for their article, but they allow anyone to download, reuse, reprint, modify, distribute, and/or copy articles in IJOCTA, so long as the original authors and source are credited. To see the complete license contents, please visit http://creativecommons.org/licenses/by/4.0/. 